\title{
Acute Delirium Associated With Levofloxacin
}

\author{
Yana Kogan ${ }^{\mathrm{a}}$, Nizar Elias ${ }^{\mathrm{a}}$, Alona Paz $^{\mathrm{b}}$, Majed Odeh ${ }^{\mathrm{a}, \mathrm{c}}$
}

\begin{abstract}
Delirium is considered as the most common complication afflicting hospitalized elderly patients, accompanied by high morbidity and mortality rate; and despite its high prevalence, it often remains unrecognized. Drug-induced delirium is a well-known entity with sedatives, narcotics and anticholinergics most often implicated in its causation. Delirium attributed to antibiotics, mainly cephalosporins and macrolids, has been infrequently reported, and until yet only seven cases of levofloxacin-induced delirium have been described in the medical literature. We describe another case of delirium associated with levofloxacin in an elderly patient who was hospitalized in our medical ward for pneumonia. The present case and the other cases previously reported should raise the awareness of physicians to this serious, underestimated, and underdiagnosed adverse effect of a commonly used antibiotic, levofloxacin.
\end{abstract}

Keywords: Levofloxacin; Quinolone; Fluoroquinolone; Delirium

\section{Introduction}

Delirium, a term used to describe an acute confusional state, is defined as a relatively acute decline in cognition that fluctuates over hours or days. The hallmark of delirium is a deficit of attention, although all cognitive domains, including memory, executive function, visuospatial tasks, and language, are variably involved. Associated symptoms may include altered sleep-awake cycles, perceptual disturbances such as hallucinations or delusions; affect changes, and autonomic findings that include heart rate and blood pressure instability [1]. It is a result of multiple interacting predisposing and precipitating factors $[1,2]$. The prevalence of delirium increases with age, and hospitalized elderly patients are particularly at risk to become acutely confused $[1,2]$. The two most consistently identified risks for delirium are older age and baseline cognitive dys-

Manuscript submitted July 10, 2018, accepted July 20, 2018

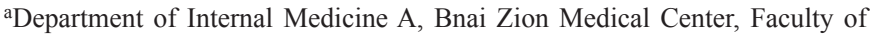
Medicine, Technion - Israel Institute of Technology, Haifa, Israel

bInfectious Diseases Unit, Bnai Zion Medical Center, Faculty of Medicine, Technion - Israel Institute of Technology, Haifa, Israel

${ }^{\mathrm{c} C}$ Corresponding Author: Majed Odeh, Department of Internal Medicine A, Bnai Zion Medical Center, Golomb Street 47, Haifa 31048, Israel.

Email: majed.odeh@b-zion.org.il

doi: https://doi.org/10.14740/jocmr3538w function [1]. It is considered as the most common complication afflicting hospitalized elderly patients; and despite its high prevalence, it often remains unrecognized, with a recent study estimating the rate of undetected delirium to be as high as $60 \%$ [3]. It can negatively affect outcomes by increasing length of stay, morbidity, and mortality, yet is often preventable and potentially reversible $[3,4]$.

Drug-induced delirium is a well-known entity with sedatives, narcotics and anticholinergics most often implicated in its causation $[1,5]$. Delirium attributed to antibiotics, mainly cephalosporins and macrolides, has been infrequently reported [6-8]. To the best of our knowledge, only seven cases of levofloxacin-induced delirium have been previously described in the medical literature [9-15], one of them from our medical ward [11]. We describe another case of delirium associated with levofloxacin in an elderly patient who was hospitalized in our medical ward for pneumonia.

\section{Case Report}

A 79-year-old woman was admitted to our medical ward for right lower lobe pneumonia accompanied by fever, non-productive cough and dyspnea for the last 3 days. Her other medical history included essential hypertension and hypercholesterolemia, for the last several years, well controlled by ramipril and simvastatin; and she was allergic to penicillin. She had no history of confusional state, and no any other documented disorder of the central nervous system (CNS).

Physical examination revealed a fully conscious and oriented woman, with normal informed bedside examination of her cognitive state. Her blood pressure was $136 / 80 \mathrm{~mm} \mathrm{Hg}$, heart rate $104 \mathrm{bpm}$, and body temperature $38.4{ }^{\circ} \mathrm{C}$. No signs of meningeal irritation were observed. Examination of the lungs revealed fine inspiratory rales and decreased ventilation over the base of the right lung, as well as, diffuse bilateral rhonchi. Laboratory examinations revealed white blood cell count (WBC) of $24,200 / \mathrm{mm}^{3}$ with $84 \%$ neutrophils, hematocrit $39 \%$, serum creatinine $0.9 \mathrm{mg} / \mathrm{dL}$, urea $40 \mathrm{mg} / \mathrm{dL}$ sodium 138 $\mathrm{mEq} / \mathrm{L}$, potassium $4.3 \mathrm{mEq} / \mathrm{L}$, C-reactive protein (CRP) 96 $\mathrm{mg} / \mathrm{L}$, arterial blood $\mathrm{O}_{2}$ saturation $90 \%$ on room air, and $\mathrm{pCO}_{2}$ $41 \mathrm{~mm} \mathrm{Hg}$. The cheat X-ray film demonstrated a right lower lobe infiltrate.

Empiric treatment with oral levofloxacin, $750 \mathrm{mg}$ daily, was started. Forty-eight h later the patient felt better, her body temperature decreased back to normal range, the heart rate and blood pressure were in the normal range, arterial blood $\mathrm{O}_{2}$ saturation increased to $96 \%$ on room air, WBC decreased to $15,100 / \mathrm{mm}^{3}$ and serum CRP level decreased to $24 \mathrm{mg} / \mathrm{L}$. On 
the third day of hospitalization the patient became confused, irritable, and disoriented to time, place, and person. She had optic and acoustic hallucinations, and her attention capacity became impaired. The patient body temperature, heart rate and blood pressure were in the normal range, no dyspnea was observed, and her arterial blood $\mathrm{O}_{2}$ saturation was $97 \%$ on room air. Neurological examination revealed no signs of meningeal irritation and no lateralizing motor findings. Serum levels of electrolytes, including sodium, potassium, magnesium, and calcium were normal. Computerized tomography (CT) scan of the brain, and electroencephalogram (EEG) performed while the patient was awake, were unremarkable.

The diagnosis of delirium was made according to the American Psychiatric Association's Diagnostic and Statistical Manual Mental Disorders (DMS IV); and was related to levofloxacin. Treatment with levofloxacin was stopped immediately after 3 days of administration, and treatment with azithromycin was started. Subsequently, the patient neurological condition gradually improved, and complete resolution of the delirium was documented $48 \mathrm{~h}$ after discontinuation of levofloxacin treatment. Upon discharge from our medical ward, she was referred to our outpatient medical clinic. Three weeks later a repeat chest X-ray film showed complete resolution of the pulmonary infiltrate, and informed bedside examination of her cognitive state was normal.

\section{Discussion}

Delirium can be related to several predisposing factors, and in our patient the pneumonia itself with its accompanying febrile illness and hypoxemia, or drugs may have been involved [2]. The delirium developed on the third day of levofloxacin treatment, and was not associated to the initial severe phase of pneumonia. It developed while the patient was afebrile, with normal heart rate, blood pressure, and arterial oxygen saturation, and with no serum electrolyte disturbances or signs of dehydration. Except of the delirium itself, the neurological examination was essentially normal, brain CT and EEG did not reveal any significant abnormality, thus excluding a primary neurological disease. However, the patient is an elderly where this risk factor might predispose to the development of delirium induced by levofloxacin.

Drug-induced delirium is a well-known entity with sedatives, narcotics and anticholinergics most often implicated in its causation [3,5]. Delirium attributed to antibiotics, mainly cephalosporins and macrolids, has been infrequently reported [6-8], and until yet only seven cases of levofloxacin-induced delirium have been described in the medical literature [9-15], one of them from our medical ward [11].

Levofloxacin is a third generation fluorinated quinolone antibiotic, the active levo stereoisomer of ofloxacin, having a broad spectrum of antibacterial activity against both grampositive and gram-negative bacteria. It belongs to the group of new quinolone derivatives (levofloxacin, sparfloxacin, grepafloxacin, trovafloxacin, gatifloxacin and moxifloxacin). As with levofloxacin, delirium has rarely been reported in association with other quinolones $[4,16-20]$.
The mechanism behind fluoroquinolones-associated delirium is still not fully understood, but has been hypothesized to involve these antibiotics having N-methyl-D-aspartate (NMDA) agonist activity, as well as, acting partly as gammaaminobutyric acid (GABA)-A receptor antagonism [4]. Flouoroquinolones may lower the seizure threshold by binding competitively to the GABA-A receptor $[21,22]$. Non-convulsive status epilepticus (NCSE) is an epileptic condition often under-diagnosed, clinically manifested by an altered mental state, and associated with continuous or recurrent epileptic form discharge on EEG [22]. NCSE associated with the use of fluoroquinolones and manifesting as an acute confusional state has been reported [23]. Since the EEG of our patient did not show features of NCSE, this possible side effect of levofloxacin can be excluded. The complete resolution of the delirium shortly after discontinuation of levofloxacin treatment supports the association of our patient's delirium to levofloxacin. Rechalenge with the drug was not done since it was considered unethical.

In conclusion, delirium is the most common complication afflicting hospitalized elderly patients, and can negatively affect outcomes by increasing length of stay at hospital, morbidity, and mortality; yet is often preventable and potentially reversible. Development of the delirium in our patient after starting treatment with levofloxacin, and its complete resolution shortly after discontinuation of the drug, in the absence of any other possible cause of delirium, supports the attribution of the delirium to levofloxacin. Although only seven cases of levofloxacin-induced delirium have been previously reported, since two cases including the present case were observed in one medical ward (ours), it indicates that this serious and potentially fatal levofloxacin-induced side effect is really underestimated and under-diagnosed world-wide. Physicians should be aware to its occurrence in order to prevent significant morbidity and mortality accompanying this serious but potentially reversible CNS complication of levofloxacin.

\section{Funding}

There was no funding support for this manuscript.

\section{Conflict of Interest}

The authors state that they have no conflict of interest.

\section{References}

1. Josephson SA, Miller BL. Confusion and Delirium. In: Kasper DL, Fauci AS, Hauser SL, Longo DL, Jameson JL, Loscalzo J, eds. Harrison's Principles of Internal Medicine. 19th ed. New York: McGraw-Hill, 2015:166-170.

2. Inouye SK. Delirium in older persons. N Engl J Med. 2006;354(11):1157-1165.

3. Hshieh TT, Inouye SK, Oh ES. Delirium in the Elderly. Psychiatr Clin North Am. 2018;41(1):1-17.

4. Sellick J, Mergenhagen K, Morris L, Feuz L, Horey A, Risbood V, Wojciechowski A, et al. Fluoroquinolone-Re- 
lated Neuropsychiatric Events in Hospitalized Veterans. Psychosomatics. 2018;59(3):259-266.

5. Brown TM. Drug-induced delirium. Semin Clin Neuropsychiatry. 2000;5(2):113-124.

6. Chow KM, Szeto CC, Hui AC, Wong TY, Li PK. Retrospective review of neurotoxicity induced by cefepime and ceftazidime. Pharmacotherapy. 2003;23(3):369-373.

7. Vicente de Vera C, Garcia M, Pifarre Teixido R, Barbe F. Delirium induced by clarithromycin in a patient with community-acquired pneumonia. Eur Respir J. 2006;28(3):671-672.

8. Mermelstein HT. Clarithromycin-induced delirium in a general hospital. Psychosomatics. 1998;39(6):540-542.

9. Hakko E, Mete B, Ozaras R, Tabak F, Ozturk R, Mert A. Levofloxacin-induced delirium. Clin Neurol Neurosurg. 2005;107(2):158-159.

10. Kiangkitiwan B, Doppalapudi A, Fonder M, Solberg K, Bohner B. Levofloxacin-induced delirium with psychotic features. Gen Hosp Psychiatry. 2008;30(4):381-383.

11. Slobodin G, Elias N, Zaygraikin N, Sheikh-Ahmad M, Sabetay S, Weller B, Odeh M. Levofloxacin-induced delirium. Neurol Sci. 2009;30(2):159-161.

12. Kocyigit I, Dortdudak S, Sipahioglu M, Unal A, Yucel $\mathrm{HE}$, Tokgoz B, Eroglu E, et al. Levofloxacin-induced delirium: is it a dangerous drug in patients with renal dysfunction? Ren Fail. 2012;34(5):634-636.

13. Lertxundi U, Palacios RH, Gutierrez FC, Domingo-Echaburu S, Garcia MG, Gomez CA. Levofloxacin-induced delirium in a patient suffering from schizoaffective disorder and multiple sclerosis. Curr Drug Saf. 2013;8(3):199200 .
14. Raj V, Murthy TV. Levofloxacin induced delirium with psychotic features in a young patient. Med J Armed Forces India. 2013;69(4):404-405.

15. Ghoshal A, Damani A, Salins N, Deodhar J, Muckaden MA. Management of levofloxacin induced anaphylaxis and acute delirium in a palliative care setting. Indian J Palliat Care. 2015;21(1):76-78.

16. McDermott JL, Gideonse N, Campbell JW. Acute delirium associated with ciprofloxacin administration in a hospitalized elderly patient. J Am Geriatr Soc. 1991;39(9):909910 .

17. Al-Ghamdi SM. Reversible encephalopathy and delirium in patients with chronic renal failure who had received ciprofloxacin. Saudi J Kidney Dis Transpl. 2002;13(2):163170.

18. Fennig S, Mauas L. Ofloxacin-induced delirium. J Clin Psychiatry. 1992;53(4):137-138.

19. Tasleem H, Viswanathan R. Moxifloxacin-induced delirium with hallucinations. Psychosomatics. 2011;52(5):472474.

20. Sumner CL, Elliott RL. Delirium associated with gatifloxacin. Psychosomatics. 2003;44(1):85-86.

21. Ball P, Mandell L, Niki Y, Tillotson G. Comparative tolerability of the newer fluoroquinolone antibacterials. Drug Saf. 1999;21(5):407-421.

22. Fernandez-Torre JL. Levofloxacin-induced delirium: Diagnostic considerations. Clin Neurol Neurosurg. 2006;108(6):614.

23. Isaacson SH, Carr J, Rowan AJ. Ciprofloxacin-induced complex partial status epilepticus manifesting as an acute confusional state. Neurology. 1993;43(8):1619-1621. 